\title{
Miocardiopatía hipertrófica familiar
}

\section{Familial hypertrophic myocardiopathy}

\author{
Marco Lazo-Soldevilla', \\ Lizeth Meza-Centeno ${ }^{2}$
}

Lazo-Soldevilla M, Meza-Centeno L. Miocardiopatía hipertrófica familiar.

Rev Soc Peru Med Interna. 2021;34(I): 31-35.

https://doi.org/10.36393/spmi.v34i1.582

\section{RESUMEN}

Presentamos el caso de un varón de 16 años de edad con el diagnóstico de miocardiopatía hipertrófica y antecedente de muerte súbita familiar, se realizó la estratificación del riesgo utilizando el modelo de predicción de riesgo clínico para pacientes con miocardiopatía hipertrófica (HCM Risk-SCD), siendo un paciente de alto riesgo por lo que se colocó un desfibrilador automático implantable como prevención primaria.

Palabras claves: miocardiopatía hipertrófica, muerte súbita cardíaca, desfibrilador automático implantable.

\section{ABSTRACT}

We present the case of a 16 year-old man with a diagnosis of hypertrophic cardiomyopathy and a history of sudden family death; risk stratification was performed using the clinical risk prediction model for patients with hypertrophic cardiomyopathy (HCM Risk -SCD), being a high-risk patient for which an implantable automatic defibrillator was placed as primary prevention.

Keywords: hypertrophic cardiomyopathy, sudden cardiac death, implantable cardioverter-defibrillator.

\section{INTRODUCCIÓN}

La miocardiopatía hipertrófica es la primera causa de muerte súbita en jóvenes por lo que en pacientes que tienen

I Médico cardiólogo. Servicio de cardiología, Hospital Nacional Ramiro Prialé Prialé, Huancayo, Junín, Perú.

2 Estudiante de medicina. Facultad de Medicina Humana, Universidad Nacional del Centro de Perú, Huancayo, Junín, Perú. una edad igual o mayor a 16 años de edad con uno o más factores de riesgo es necesario estimar el riesgo de muerte súbita cardiaca (MSC) a cinco años con el fin de evitar eventos cardiovasculares fatales a futuro con la colocación de un DAI. ${ }^{1}$

\section{PRESENTACIÓN DEL CASO}

Paciente varón de 16 años de edad, nacido en Perú, con historia familiar de muerte súbita temprana de hermano mayor debido a miocardiopatía hipertrófica $(\mathrm{MCH})$ y padre en control con el mismo diagnóstico. Cursa con disnea progresiva y palpitaciones de tres años de evolución. Al examen físico: despierto, lucido, Glasgow 15, PA 90/60 $\mathrm{mm} \mathrm{Hg}$, FC 60 por minuto, FR 18 por minuto. Resto del examen sin alteraciones.

Electrocardiograma (ECG) con signos de hipertrofia y sobrecarga ventricular izquierda (Figura 1)

Ecocardiografía transtorácica: engrosamiento del septum interventricular con un grosor máximo de $30 \mathrm{~mm}$, gradiente del tracto de salida del ventrículo izquierdo en $47 \mathrm{~mm} \mathrm{Hg}$ (Figuras 2 y 3), movimiento sistólico anterior (SAM) de la válvula mitral, insuficiencia mitral moderada, 


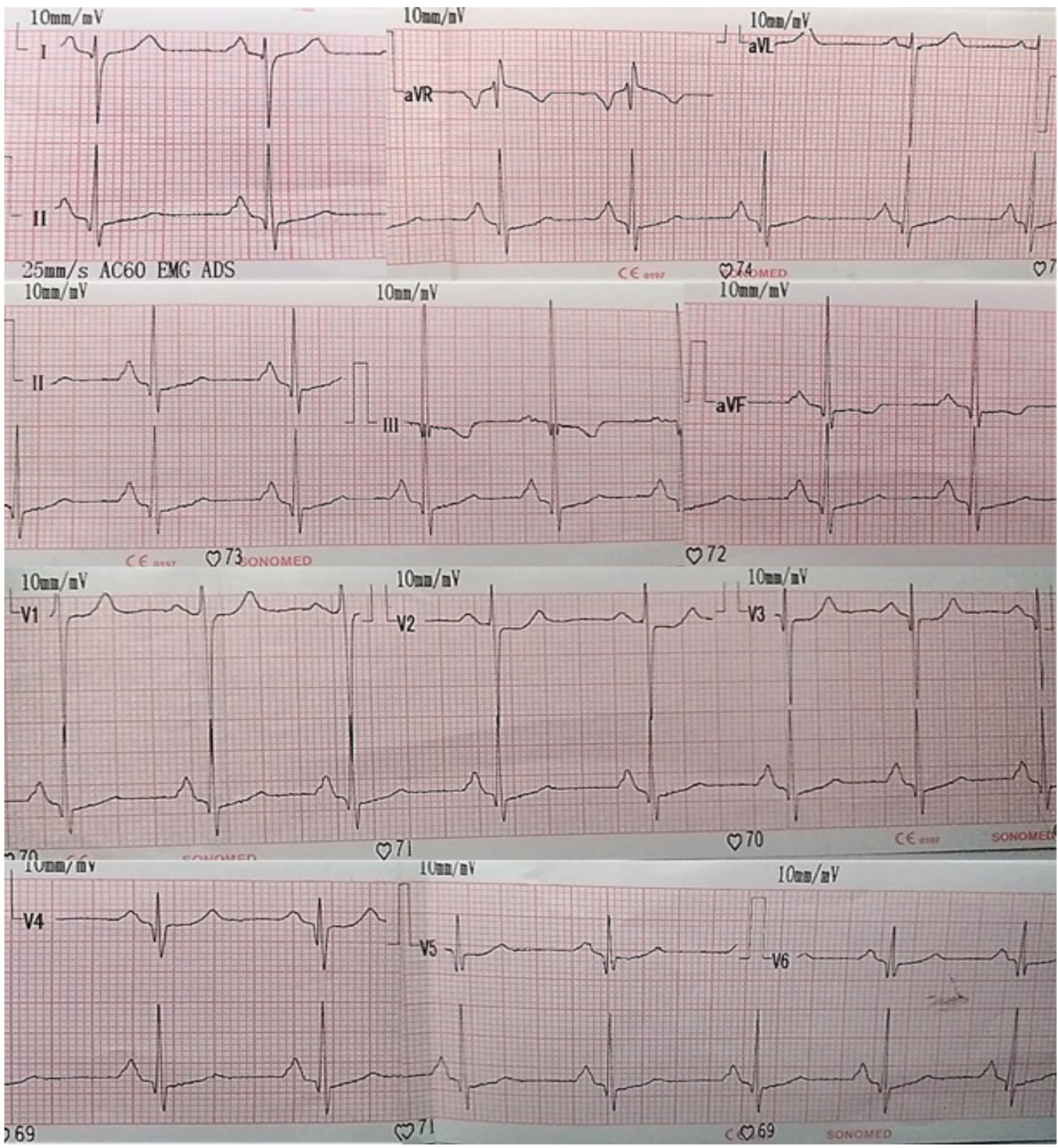

Figura I. ECG: Ritmo sinusal, signos de hipertrofia y sobrecarga ventricular izquierda.

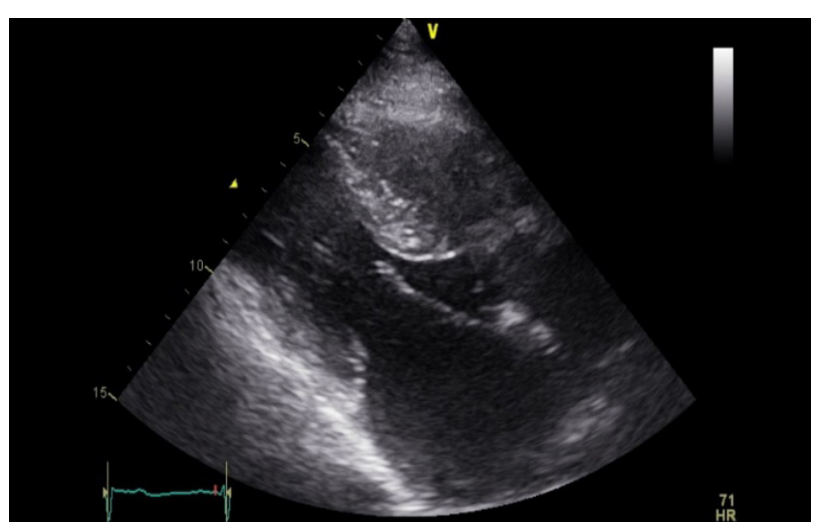

Figura 2. Engrosamiento del septum interventricular (SIV), dilatación de aurícula izquierda y movimiento sistólico anterior (SAM) de valva anterior de la válvula mitral.

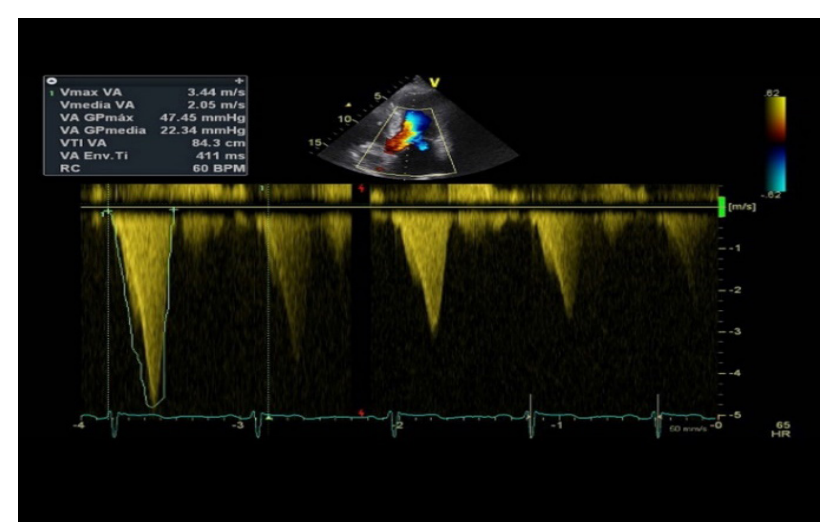

Figura 3. Gradiente del TSVI en $47 \mathrm{~mm} \mathrm{Hg}$. 
dilatación severa de la aurícula izquierda (Figuras 4a y 4b), disfunción diastólica grado III (Figura 5), función sistólica biventricular preservada y Strain Rate de -19 (Figura 6). Holter de 24 horas: ritmo sinusal, contracciones prematuras supraventriculares de baja densidad.
La cardiorresonancia evidenció: hipertrofia asimétrica con engrosamiento a predominio de pared media inferolateral y en puntos de inserción superior e inferior del VD; captación de gadolinio en realce tardío con patrón de distribución parcheado en zonas de máxima hipertrofia, asociado a realce

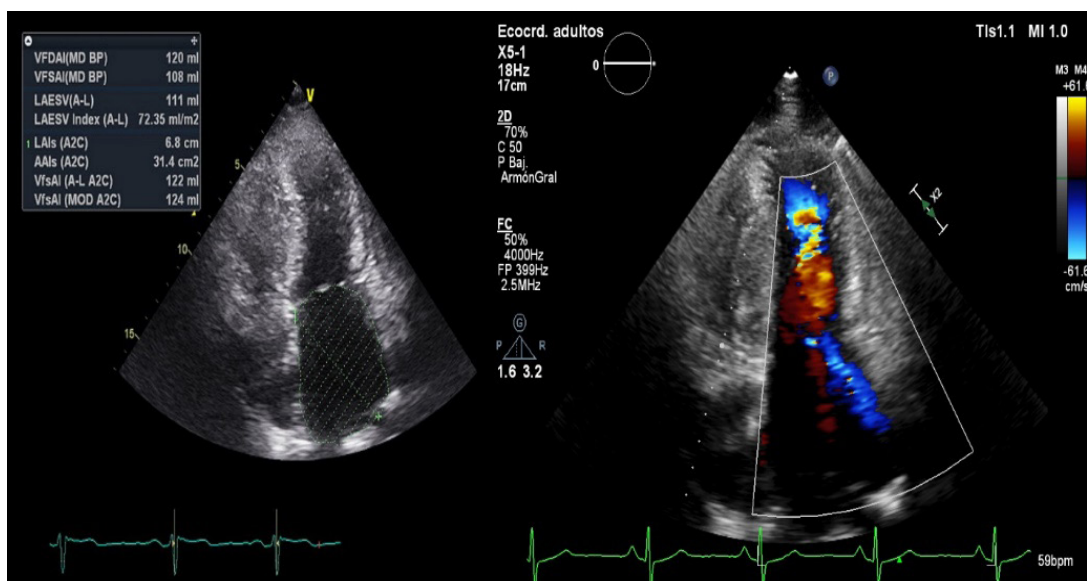

Figura 4. a. Dilatación severa de la aurícula izquierda.b- Flujo de regurgitación mitral moderada.
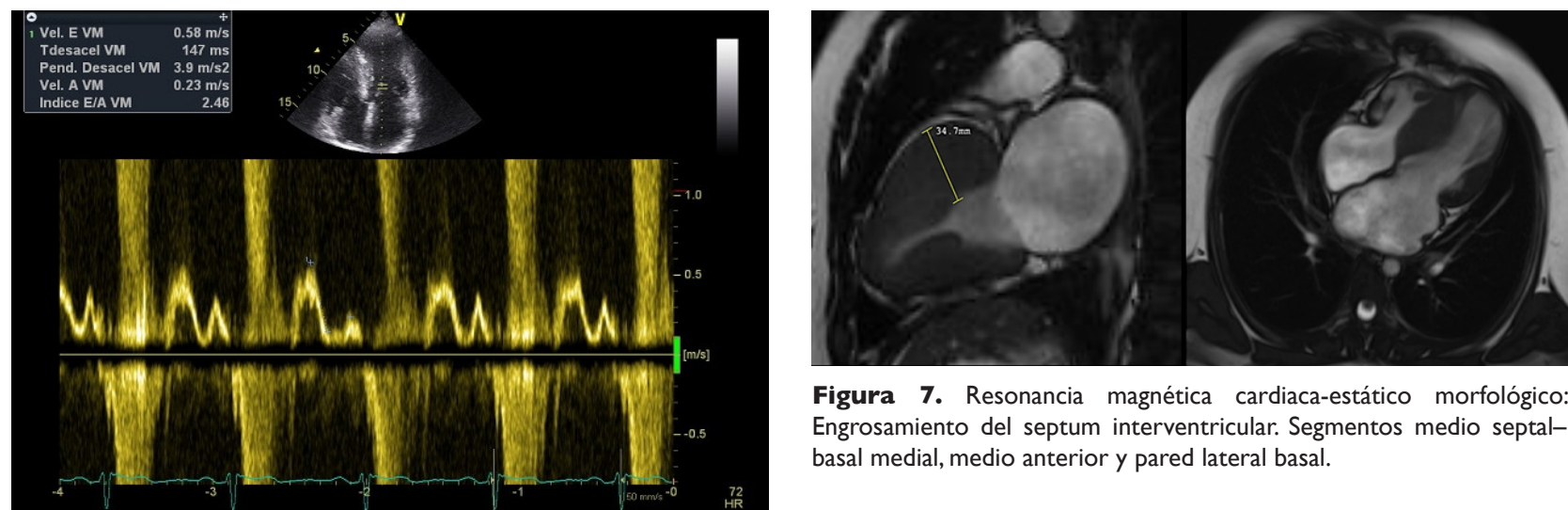

Figura 7. Resonancia magnética cardiaca-estático morfológico: Engrosamiento del septum interventricular. Segmentos medio septalbasal medial, medio anterior y pared lateral basal.

Figura 5. Patrón de relajación del VI. Disfunción diastólica grado III (severa), patrón restrictivo.
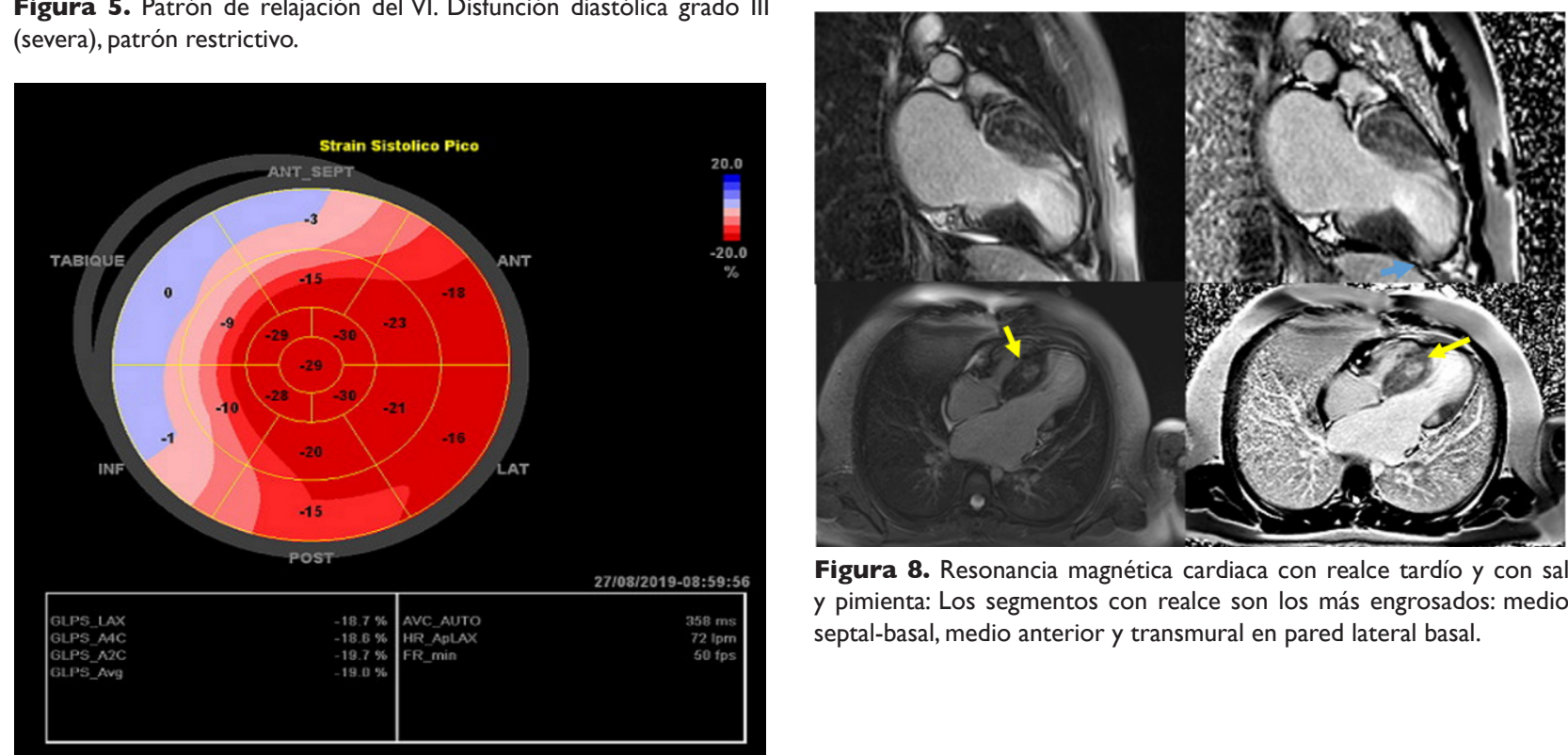

Figura 8. Resonancia magnética cardiaca con realce tardío y con sal y pimienta: Los segmentos con realce son los más engrosados: medio septal-basal, medio anterior y transmural en pared lateral basal.

Figura 6. Strain Rate - Ojo de Buey con Strain Rate de -19. 
transmural a nivel basal anterolateral y adelgazamiento de esta zona y a nivel apical (Figuras 7-9).

Se estratificó el riesgo de muerte súbita a través del score HCM Risk-SCD siendo 8,63\%, de riesgo elevado, colocándose desfibrilador automático implantable como prevención primaria.

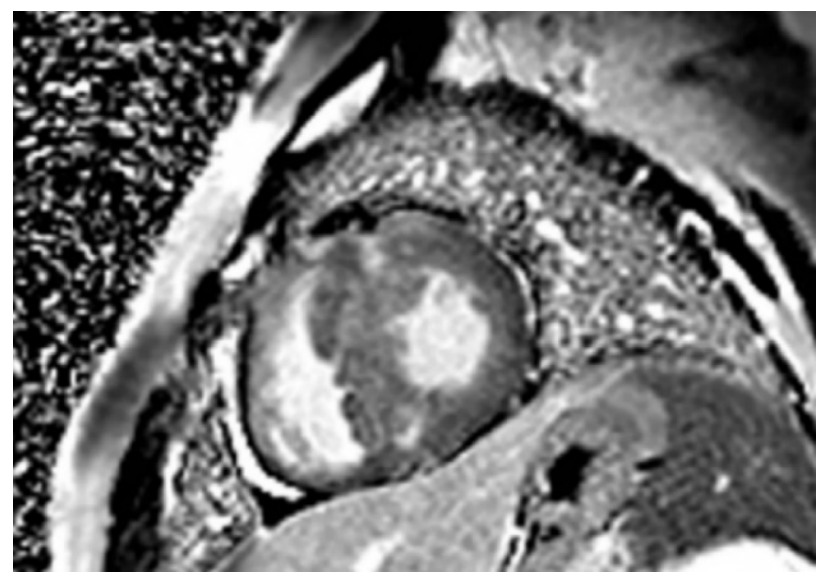

Figura 9. Resonancia magnética cardiaca con realce tardío

\section{DISCUSIÓN}

La miocardiopatía hipertrófica $(\mathrm{MCH})$ es la enfermedad cardiovascular genética más frecuente, debido a la mutación de proteínas sarcoméricas constituyendo la primera causa de muerte súbita en menores de 35 años; presenta un patrón autosómico dominante, encontrándose en uno de cada 500 de la población en general asociado a una expresion fenotipica heterogénea con un predominio en varones y una incidencia anual del 0,3 al 0,5 por 1000 personas. $^{2}$

El diagnóstico se basa en la sospecha clínica (la mayoría asintomáticos, aunque algunos presentan intolerancia al ejercicio, disnea de esfuerzo, síncope, palpitaciones o angina) asociada a una historia familiar de muerte súbita por $\mathrm{MCH}$, detección de anormalidades electrocardiográficas y confirmada con el uso de técnicas por imagen como la ecocardiografía, tomografía o cardiorresonancia que demuestran el aumento del grosor de la pared del ventrículo izquierdo (VI) $\geq 15 \mathrm{~mm}$, no explicada por una causa secundaria; en el caso de pacientes pediátricos requiere un grosor de la pared del VI de más de dos desviaciones estándar mayor que la media de la población (z-score $>2){ }^{3}$

El diagnóstico clínico en familiares de primer grado de pacientes con $\mathrm{MCH}$, se basa en la presencia de un aumento de espesor de la pared del VI $\geq 13 \mathrm{~mm}$ en uno o más segmentos miocárdicos del VI, de manera inexplicable y medido con cualquier técnica por imagen cardíaca. ${ }^{4}$

El electrocardiograma muestra signos de sobrecarga e hipertrofia del VI, ondas Q patológicas y anormalidades en el segmento ST u onda T. Este se debe realizar desde el primer control de una $\mathrm{MCH}$ conocida y si hay cambios en los síntomas del paciente; se considera un marcador precoz sensible de esta enfermedad en los familiares. ${ }^{4}$

La ecocardiografía transtorácica es considerada como primer método de estudio ante la sospecha de $\mathrm{MCH}$. Esta evaluación permite detectar la distribución y el grosor máximo de hipertrofia del VI, siendo este $\geq 30 \mathrm{~mm}$ un factor de riesgo de muerte súbita al igual que la obstrucción del tracto de salida del VI definida por un gradiente $\geq 30$ $\mathrm{mm} \mathrm{Hg}$ en reposo o durante la maniobra de Valsalva y dilatación auricular izquierda. Así mismo, permite valorar el movimiento sistólico anterior (SAM) del velo anterior de la válvula mitral, como causa de obstrucción dinámica del TSVI e insuficiencia mitral; la función sistólica del VI; la disfunción diastólica del VI, que tiene valor pronóstico en la aparición de síntomas, tolerabilidad al ejercicio, falla cardiaca y muerte. Además, valora el volumen auricular izquierdo, la presión de la arteria pulmonar y el Strain Rate que permite diferenciar la $\mathrm{MCH}$ del engrosamiento inducido por hipertensión arterial, miocardiopatías infiltrativas y por depósito; en el caso de MCH hay una disminución del Strain longitudinal y el Strain circunferencial reforzado de acuerdo a la extensión de la hipertrofia y mala disposición de las fibras miocárdicas. ${ }^{4}$

La ecocardiografía transesofágica (ETE) es útil para definir el mecanismo de obstrucción del TSVI, evaluar el aparato de la válvula mitral a nivel pre y perioperatorio para guiar la cirugía y detectar complicaciones en la miectomía septal, y ante la sospecha de insuficiencia mitral severa. La ETE con inyección intracoronaria de contraste de las arterias perforantes septales guía la ablación septal con alcohol cuando las ventanas transtorácicas son insuficientes para visualizar el contraste intracardiaco. ${ }^{5}$

La resonancia cardiaca es el patrón de oro para la evaluación del grosor del VI y la medición de los volúmenes cardiacos; técnica de imagen complementaria cuando la ventana ecocardiográfica es inadecuada, que permite estratificar el riesgo de muerte súbita cuando el grosor de la pared del VI es $\geq 30 \mathrm{~mm}$; evalúa el realce tardío con gadolinio con un patrón irregular o parcheado en áreas de hipertrofia y en los puntos de inserción superior e inferior del VD; e identifica fibrosis miocárdica, marcador pronostico en la $\mathrm{MCH}$ que permite diferenciar esta de otras miocardiopatías como la amiloidosis y la enfermedad de Fabry; y la secuencia cine de fase de contraste que permite valorar la morfología de la $\mathrm{MCH}$, su distribución y la anatomía del aparato valvular mitral y de los músculos papilares para establecer la mejor terapia invasiva. ${ }^{4}$

La biopsia endomiocárdica puede considerarse ante la sospecha de miocardiopatía infiltrativa o por almacenamiento, después de la biopsia de otros tejidos más accesibles. $^{5}$

Se procedió a estratificar el riesgo de muerte súbita del paciente, cumpliéndose con los criterios mayores alcanzando una puntuación de $8,63 \%$, catalogándose como alto riesgo de muerte súbita e indicativo del implante de DAI como medida de prevención primaria. ${ }^{6}$

El tratamiento de la obstrucción del TSVI sintomática puede ser farmacológico con betabloqueadores no vasodilatadores, 
tipo bisoprolol, y como complemento a la disopiramida; o, invasivo como la miectomía septal (procedimiento de Morrow) si el gradiente es $\geq 50 \mathrm{~mm} \mathrm{Hg}$, presenta síntomas moderados a severos (NYHA Clase III-IV) y/o síncope de esfuerzo recurrente a pesar de la tolerancia máxima al tratamiento farmacológico. Una alternativa es la ablación septal, que es menos efectiva en pacientes con cicatrización septal extensa por cardiorresonancia y en pacientes con hipertrofia muy severa $(\geq 30 \mathrm{~mm}){ }^{5}$

La guia de miocardiopatia hipertrofica de la sociedad europea de cardiología 2014 recomienda estratificar el riesgo de muerte súbita a todo paciente con el diagnóstico de MCH utilizando el score HCM Risk-SCD que se basa en los siguientes factores de riesgo mayor para muerte súbita: edad temprana al momento del diagnóstico, miocardiopatía hipertrófica obstructiva (gradiente del tracto de salida del ventrículo izquierdo $\geq 30 \mathrm{~mm} \mathrm{Hg}$ o un $\mathrm{z}$-score $\geq 6$, grosor maximo del VI $\geq 30 \mathrm{~mm}$ o un $z$-score $\geq 6$, síncope de causa desconocida, taquicardia ventricular no sostenida y la historia familiar de MSC; considerándose $>6 \%$ de alto riesgo, indicativo de colocación de un cardiodesfibrilador implantable (DAI), su importancia se debe a que 1 por cada 16 DAI implantados en pacientes con $\geq 4 \%$ de riesgo de MSC a 5 años, se salvará de MSC. ${ }^{6}$

El diagnóstico diferencial se establece con hipertrofia del VI debido a hipertensión arterial, estenosis aortica o enfermedad renal crónica; así mismo con la amiloidosis, el corazón de atleta, miocardiopatías infiltrativas como la enfermedad de Pompe o Anderson-Fabry, y enfermedades metabólicas como el síndrome de Hunter. ${ }^{5}$

La ecocardiografía del corazón de atleta, muestra una hipertrofia excéntrica biventricular con un grosor de pared inferior a $15 \mathrm{~mm}$ y un ventrículo izquierdo moderadamente dilatado, a diferencia de la $\mathrm{MCH}$ que se caracteriza por hipertrofia ventricular izquierda asimétrica con un diámetro del VI reducido. En hasta el 70\% de los casos, la obstrucción del tracto de salida del ventrículo izquierdo es evidente durante la ecocardiografía de estrés. ${ }^{5}$

También se recomienda el estudio genético y consejería genética previo y posterior a las pruebas tanto del paciente y de sus familiares.

En conclusión, el caso reporta la importancia de reconocer de manera oportuna una miocardiopatía de presentación familiar, la estratificación de riesgo de muerte súbita con el score HCM Risk-SCD con el fin de tomar medidas de prevención primaria a través de la colocación de un desfibrilador automático Implantable (DAI) y alternativas invasivas y quirúrgicas en su tratamiento.

\section{REFERENCIAS BIBLIOGRÁFICAS}

I. Ommen SR, Mital S, Burke MA, Day SM, Deswal A, Elliott P, et al. 2020 AHA/ACC Guideline for the diagnosis and treatment of patients with hypertrophic cardiomyopathy: A report of the American College of Cardiology/American Heart Association Joint Committee on Clinical Practice Guidelines. J Am Coll Cardiol. 2020 Nov 20.

2. Liew AC, Vassiliou VS, Cooper R, Claire RE. Hypertrophic cardiomyopathy-past, present and future.J Clin Med. 2017 December; 6(12): p. 118

3. Ullal AJ, Abdelfattah RS, Ashley EA, Froelicher VF. Hypertrophic cardiomyopathy as a cause of sudden cardiac death in the young: $A$ meta-analysis. Am J Med. 2016 May; I29(5): p. 486-496.

4. Maron BJ, Maron MS. The remarkable 50 years of imaging in HCM and how it has changed diagnosis and management from M-mode echocardiography to CMR. JACC: cardiovascular imaging. 2016 November; 9(7): p. 858-872.

5. Elliott PM,Anastasakis A, Borger MA, Borggrefe M, Cecchi F, Charron P, et al. Guía de práctica clínica de la ESC 2014 sobre el diagnóstico y manejo. Rev Esp Cardiol. 2015 July; 68(I): p. 63.el-e52.

6. O'Mahony C, Jichi F, Pavlou M, Monserrat L, Anastasakis A, Rapezzi $C$, et al. A novel clinical risk prediction model for sudden cardiac death in hypertrophic cardiomyopathy (HCM Risk-SCD). Eur Heart J. 2013 August 7; 35(30): p. 2010-2020.

\section{CORRESPONDENCIA \\ Lizeth Meza Centeno \\ lizethkmc@hotmail.com}

Fecha de recepción: 15-12-2020.

Fecha de aceptación: |0-0|-202I. 